\title{
Bulk-Explosion-Induced Metal Spattering During Laser Processing
}

\author{
Cang Zhao, ${ }^{1}$ Qilin Guo, ${ }^{2}$ Xuxiao Li,${ }^{3}$ Niranjan Parab, ${ }^{1}$ Kamel Fezzaa, ${ }^{1}$ Wenda Tan, ${ }^{3, *}$ Lianyi Chen,,${ }^{2, \dagger}$ and Tao Sun ${ }^{1, *}$ \\ ${ }^{1}$ X-ray Science Division, Argonne National Laboratory, Lemont, Illinois 60439, USA \\ ${ }^{2}$ Department of Mechanical and Aerospace Engineering, Missouri University of Science and Technology, \\ Rolla, Missouri 65409, USA \\ ${ }^{3}$ Department of Mechanical Engineering, University of Utah, Salt Lake City, Utah 84112, USA
}

(Received 11 December 2018; revised manuscript received 7 April 2019; published 14 June 2019)

\begin{abstract}
Spattering has been a problem in metal processing involving high-power lasers, like laser welding, machining, and recently, additive manufacturing. Limited by the capabilities of in situ diagnostic techniques, typically imaging with visible light or laboratory x-ray sources, a comprehensive understanding of the laser-spattering phenomenon, particularly the extremely fast spatters, has not been achieved yet. Here, using MHz single-pulse synchrotron-x-ray imaging, we probe the spattering behavior of Ti-6Al-4V with micrometer spatial resolution and subnanosecond temporal resolution. Combining direct experimental observations, quantitative image analysis, as well as numerical simulations, our study unravels a novel mechanism of laser spattering: The bulk explosion of a tonguelike protrusion forming on the front keyhole wall leads to the ligamentation of molten metal at the keyhole rims and the subsequent spattering. Our study confirms the critical role of melt and vapor flow in the laser-spattering process and opens a door to manufacturing spatter- and defect-free metal parts via precise control of keyhole dynamics.
\end{abstract}

DOI: 10.1103/PhysRevX.9.021052

Subject Areas: Fluid Dynamics, Materials Science, Mechanics

\section{INTRODUCTION}

Laser spattering refers to the ejections of molten metal from a pool heated by a high-power stationary or scanning laser [1,2]. Generally, spattering is a highly undesired phenomenon in laser processing and manufacturing. In laser welding, when the spatters resolidify around the periphery of a weld seam, the necessary postremoval process (typically involving grinding, polishing, or abrasive blasting) will not only alter the original part morphology but also increase the production time and cost [3,4]. In laser-powder-bed-fusion additive manufacturing, spattering could trigger a chain reaction of negative consequences. When the laser beam scans across the powder bed, spatters may get ejected near the rims of vapor depression (i.e., keyhole) [5]. When they collide with each other or with the cold raw powders, those blown away by the metal vapor as shown in the Supplemental Material Video S1 [6], larger and irregular agglomerations can then form via

\footnotetext{
*wenda.tan@mech.utah.edu

†chenliany@mst.edu

taosun@aps.anl.gov
}

Published by the American Physical Society under the terms of the Creative Commons Attribution 4.0 International license. Further distribution of this work must maintain attribution to the author(s) and the published article's title, journal citation, and DOI. particle-particle coalescence or sintering [7]. These particles tend to have different compositions, microstructures, and morphologies from the original feedstock [8-10], creating problems for powder recycling. Also, when they fall back onto the powder bed, they will negatively affect the powder recoating, resulting in the formation of structural defects (e.g., lack-of-fusion porosity) and the degradation of mechanical properties (e.g., fatigue life) in the end products $[11,12]$.

Over the last few decades, the research community, through its endeavors, has been expanding our knowledge of the formation, development, and controls of laser spattering [13,14]. A few mechanisms were proposed to explain the laser-spattering phenomenon, which are mostly concerned with the local laser energy absorption, vapor plume dynamics, and melt flow $[15,16]$. The complex interplays among recoil pressure, vapor plume impact, and the surface tension of the molten metal are believed to be the cause of spattering. However, because of the highly dynamic and transient nature of laser-matter interactions, the key physical processes that lead to the initial formation of spatters have not been completely captured, primarily held back by the limitations of in situ monitoring techniques. Also, the explanation of the occurrence of extremely fast spatters (i.e., $>40 \mathrm{~m} / \mathrm{s}$ in the case of Ti-6Al-4V) is still vague and unclear. While high-speed visible-light and thermal imaging techniques allow the observation of the surface feature evolutions [17-20], the 
subsurface information (i.e., keyhole fluctuations and melt pool dynamics) are largely unknown and often subject to speculation. Laboratory $\mathrm{x}$-ray sources have been used for detecting the subsurface structural changes [21,22]; however, the limited $\mathrm{x}$-ray flux yields low imaging resolutions in both space and time domains.

To address these issues, here we present $\mathrm{MHz}$ singlepulse $\mathrm{x}$-ray imaging results obtained at a third-generation high-energy synchrotron facility. A novel mechanism for laser spattering is discovered in our experiment. By combining with image analysis and numerical simulations, we unravel that the bulk explosion of a tonguelike protrusion forming on the front keyhole wall induces the ligamentation of the molten metal at the keyhole rims and the subsequent spattering of extremely fast droplets. The high penetration power of hard $\mathrm{x}$ rays and high resolutions of the imaging technique enable us, for the first time ever, to connect the spattering behavior above the surface with the keyhole dynamics below the surface and inside the sample.

This paper proceeds as follows. In Sec. II A, we first present the $\mathrm{MHz}$ full-field $\mathrm{x}$-ray image series of a representative laser-spattering process, from which we extract and summarize four common sequential events that lead to spattering: (i) transformation of the keyhole morphology from a letter "J"-like shape to a reverse-triangle-like shape, (ii) formation and evolution of a tonguelike protrusion from the front keyhole wall, (iii) local curvature changes around the keyhole rims, and (iv) formation and evolution of melt ligaments and spatters. Next, based on quantitative image analysis as well as numerical modeling, we present detailed discussions in Secs. II B and II C on the physical processes underpinning these four events. In Sec. II B, we explain the mechanism that is responsible for the occurrence of the bulk explosion. In Sec. II C, we study the motions of melt ligaments and spatters, and in particular, we connect the spatter-ejection behavior with the structural dynamics of the front keyhole wall. In Sec. III, we conclude our findings and discuss perspectives for future research and practical implementations to mitigate spattering. Further details about the experimental method, quantitative analysis, as well as numerical modeling are provided in the Appendixes and the Supplemental Material [6].

\section{EXPERIMENTAL RESULTS}

\section{A. MHz synchrotron-x-ray imaging of laser-induced spattering}

The spattering behavior of Ti-6Al-4V in the scanninglaser-melting process is captured using $\mathrm{MHz} \mathrm{x}$-ray imaging at the 32-ID-B beam line of the Advanced Photon Source (APS). The experimental schematic is shown in Fig. S1 of the Supplemental Material [6], with the details in Appendix A [7,23,24]. Figure 1 and Supplemental Material Fig. S2 [6] show the dynamic $\mathrm{x}$-ray image series of two representative spattering processes. Frame-by-frame images $(1.087 \mathrm{MHz})$ as well as schematic illustrations are documented in the Supplemental Material Figs. S3 and S4 and Video S2 [6]. In each spattering process, four common sequential events can be extracted, referred to as events nos. 01-04. In addition, the appearance and vanishing of a keyhole pore (KP) is found in Fig. 1, and we refer to it as event KP.

\section{Four common events in laser-induced spattering}

Event no. 01 refers to small protrusions forming on the front keyhole wall, accompanied by the transformation of the keyhole morphology. As shown in the first few frames of Fig. 1, a small protrusion appears at the front keyhole wall near the rim and then flows down towards the keyhole bottom. After a few such small protrusions, the keyhole changes from a J-like shape to a reverse-triangle-like shape. The formation of small protrusions and the $\mathrm{J}$-shaped keyhole have been discussed previously by other researchers [25], whereas the keyhole morphology change to the reversetriangle-like shape is a highly transient process, which is first observed here using a $\mathrm{MHz} \mathrm{x}$-ray imaging technique.

Event no. 02 refers to the formation and evolution of a tonguelike protrusion from the front keyhole wall. As revealed in our X-ray images, following the small protrusions in event no. 01, at $135.24 \mu \mathrm{s}$, a second protrusion forms at the front keyhole wall rim and then flows down. Abruptly, at $136.16 \mu \mathrm{s}$, the motion of the protrusion slows down, and its shape transforms from the original dome leaning downward to a small rod tilting upward. The protrusion grows rapidly, with a deep and narrow minikeyhole on its top and then stretches rapidly towards the horizontal center of the keyhole, forming a tonguelike protrusion at $137.08 \mu \mathrm{s}$, which disappears within a microsecond, indicating an explosionlike collapse. Almost at the same time, a chaotic pattern containing subresolution features is shown in the keyhole. The remains of the protrusion (i.e., the root part connecting with the front keyhole wall) moves down and disappears at the keyhole bottom. Unlike the small protrusions in event no. 01, this tonguelike protrusion has never been observed and reported by others before.

Events no. 03 and no. 04, which take place simultaneously, describe the local curvature change of the keyhole walls and the morphology change of the melt around the top keyhole rims, respectively. In event no. 03, immediately after the dramatic collapse of the tonguelike protrusion described in event no. 02 , the local curvatures around the keyhole rims undergo rapid changes. Meanwhile, the rear keyhole wall near the rim contains random roughness and wrinkles, while the front keyhole wall is relatively smooth. In event no. 04, thin melt ligaments emerge from the rims (above or below the sample surface), then rise, neck, and break up into spatters in the end.

\section{A separate event showing an instant keyhole pore}

As shown in Fig. 1, a separate event KP discloses the details of the formation and evolution of an instant keyhole 

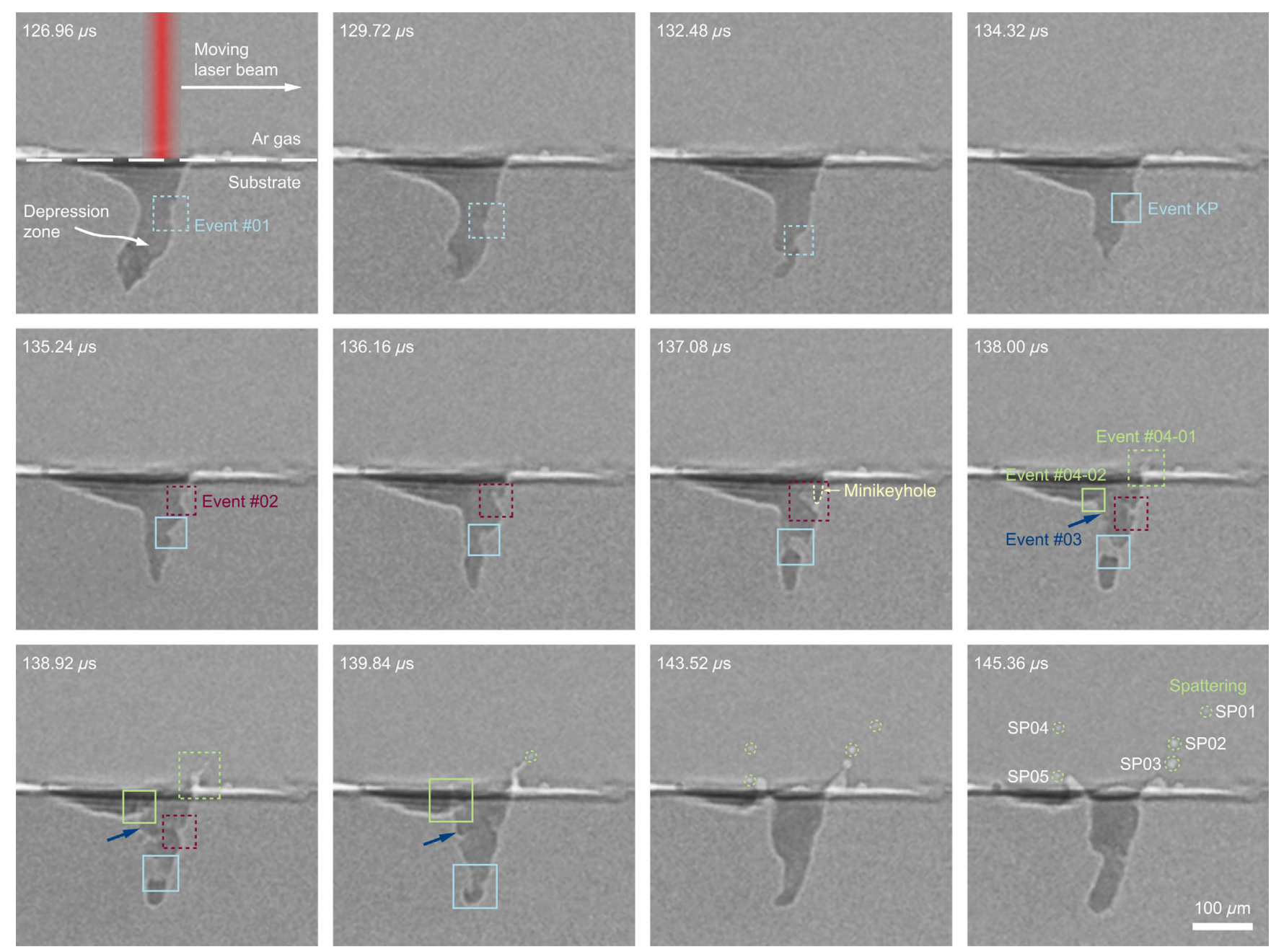

FIG. 1. MHz x-ray images of metal spattering of Ti-6Al-4V during laser processing. Four events can be extracted. Event no. 01 (sky blue dashed rectangles): A protrusion forms at the top surface and runs down along the front keyhole wall, accompanied by the keyhole morphology changing from a J-like shape to a reverse-triangle-like shape. Event no. 02 (purple dashed rectangles): A following protrusion appears, grows, and collapses around the horizontal center of the keyhole. A minikeyhole on top of the protrusion is outlined by a light yellow dashed curve. Event no. 03 (dark blue arrows): The local curvature on the rear keyhole wall changes. Event no. 04 (light green dashed and solid rectangles): Melt ligaments form, elongate, and break up into spatters (light green dashed circles numbered SP01-SP05). A separate event KP (sky blue solid rectangles) describes the formation and vanishing of a keyhole pore. The laser beam scans from left to right, with spot size of approximately $80 \mu \mathrm{m}\left(1 / e^{2}\right)$, power of $210 \mathrm{~W}$, and scanning speed of $500 \mathrm{~mm} / \mathrm{s}$. The imaging frame rate is $1.087 \times 10^{6}$ frames per second, synchronized with the $\mathrm{x}$-ray pulses. Each individual image is generated by a single $\mathrm{x}$-ray pulse (pulse width approximately $100 \mathrm{ps).} \mathrm{All} \mathrm{images} \mathrm{shown} \mathrm{here} \mathrm{are} \mathrm{background} \mathrm{corrected} \mathrm{using} \mathrm{the} \mathrm{images} \mathrm{collected} \mathrm{before} \mathrm{the} \mathrm{laser}$ melting. The contrast is then reversed to highlight the events around the keyhole. Frame-by-frame images as well as schematic illustrations are documented in the Supplemental Material Figs. S3 and S4 and Video S2 [6].

pore. When the protrusion near the keyhole bottom from the front keyhole wall collides with the rear keyhole wall, the closure of the melt traps a pore at the keyhole bottom. When the melt liquid above the pore is drilled through by the continuous laser heating, the keyhole pore escapes.

\section{B. Physical processes underpinning melt ligamentation}

In this section, we present detailed discussions on the physical processes involved in the melt ligamentation. In particular, we elaborate on the underlying mechanism. The four common events extracted from the x-ray video are schematically drawn in Fig. 2(a), where three time nodes are defined: $t_{1}$ for the formation of a reverse-triangle-like keyhole, $t_{2}$ for the formation of a tonguelike protrusion, and $t_{3}$ for the formation of melt ligaments.

\section{Formation of a reverse-triangle-like keyhole}

As illustrated in Figs. 2(a) and 2(b), when the scanning laser beam advances forward, most of the incident beam impinges on the front keyhole wall [26]. On the sample surface, the solid phase just ahead of the front keyhole rim is first heated up and melted. Once the surface temperature 
(a)
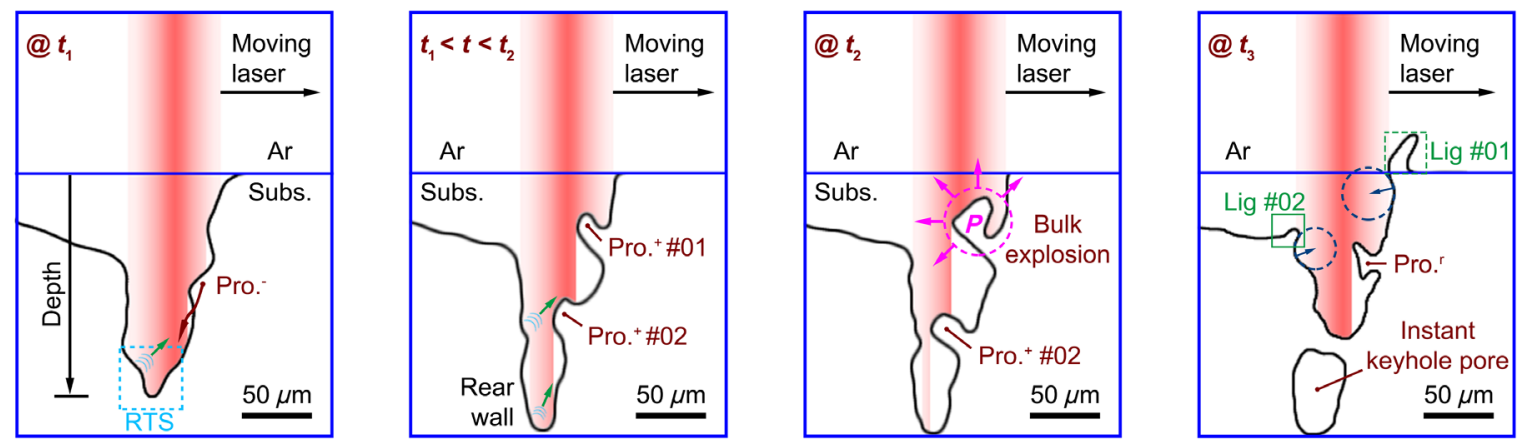

(b)

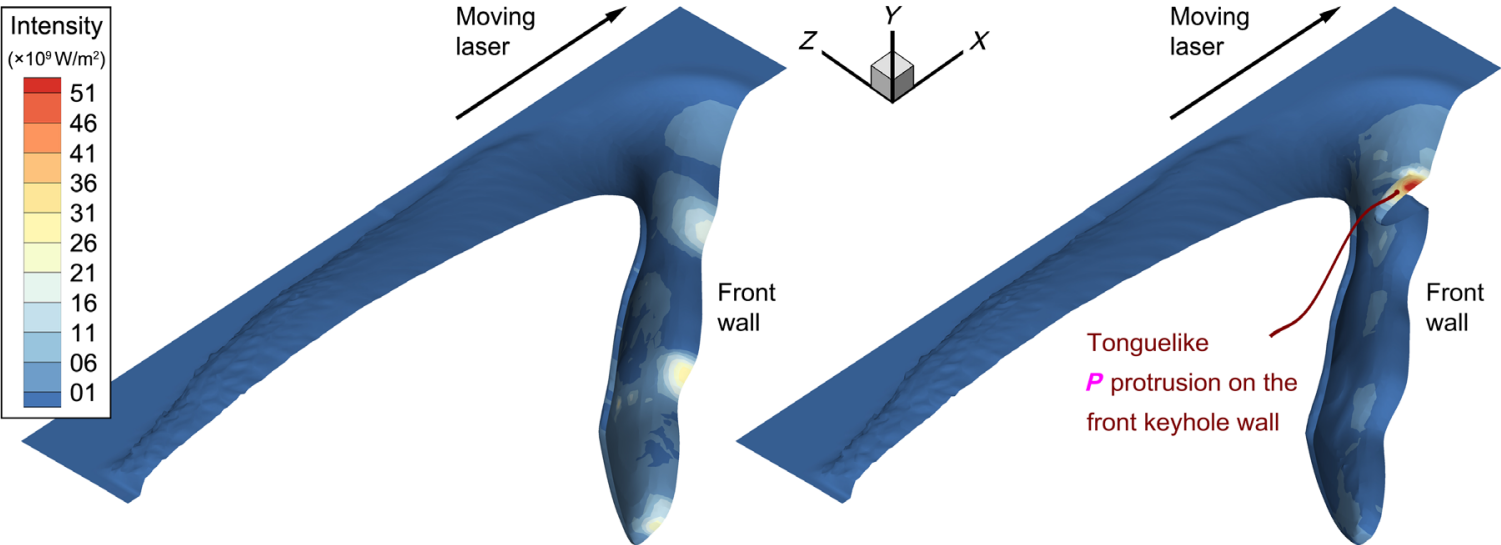

(c)

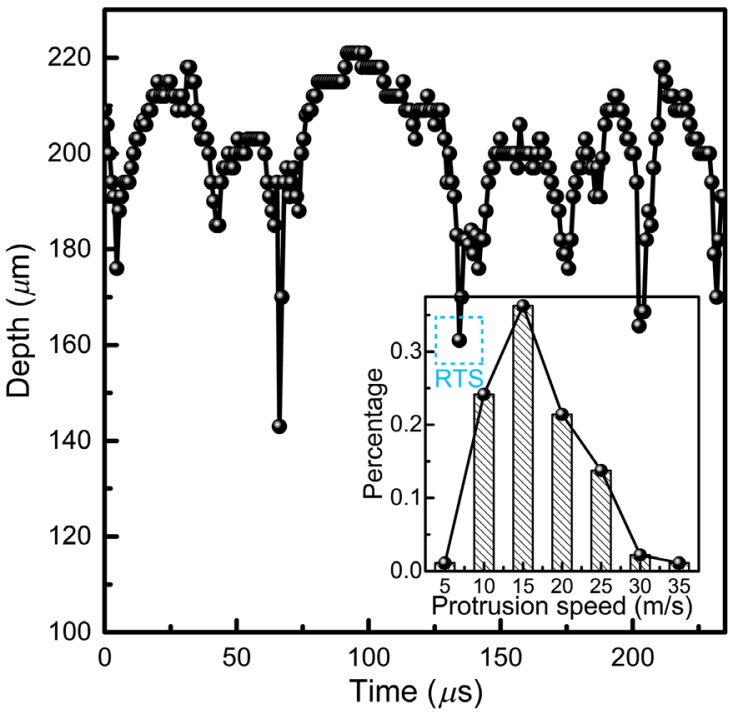

(d)

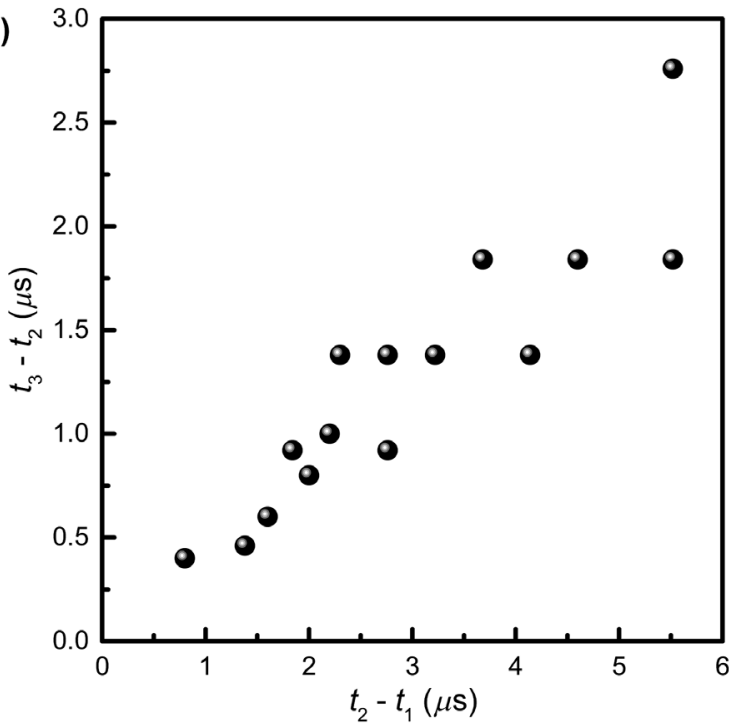

FIG. 2. Bulk explosion inside a keyhole. (a) Schematic illustrations of the sequential events that lead to melt ligamentation. At $t_{1}$, a keyhole of reverse-triangle-like shape (RTS, marked using sky blue dashed rectangle) forms. The rear wall of this unique keyhole is directly exposed to the incident laser beam, and the generated vapor plume (olive green arrows) travels upwards towards the front keyhole wall. Upon impact, the existing protrusions (e.g., Pro. no. 01 and Pro. no. 02) transform from a dome shape slightly leaning downward (denoted by Pro-) to a rod shape tilting upward (denoted by $\mathrm{Pro}^{+}$). At $t_{2}$, a tonguelike protrusion $(P)$ forms around the horizontal center of the keyhole with a minikeyhole on its top surface. The bulk explosion of the tonguelike protrusion (purple dashed circle and arrows) releases a mixture of gas and fine droplets. At $t_{3}$, the high-momentum mixture reaches the rims of the keyhole. Simultaneously, the local curvatures (dark blue dashed circles) of the keyhole walls change, and thin melt ligaments (Lig, olive green dashed and solid rectangles) appear. After the explosion, the remains of $P$ protrusion $\left(\operatorname{Pro}^{r}\right.$ ) continue to flow down and quickly vanish. The last panel shows an instant keyhole pore. (b) Simulation of laser absorption intensity with and without the tonguelike protrusion via ray tracing. (c) Keyhole depth as a function of time. Most local minima below a depth threshold correlate with the formation of reverse-triangle-like keyholes. The point marked by the sky blue dashed rectangle is the case shown here. The inset in (c) shows the distribution of the downflow speeds of the protrusions on the front keyhole wall, with the sampling volume of 182. (d) Relationship between the action time of the tonguelike protrusion $t_{3}-t_{2}$ and its formation time $t_{2}-t_{1}$. Data points in this plot are collected from $\mathrm{x}$-ray videos (frame rate varies from 1.087 to $5 \mathrm{MHz}$ ) of multiple spattering processes. 
of the molten metal reaches its boiling point, local evaporation happens, which generates a recoil on the liquid below. A small protrusion is then formed due to the local vapor depression. Propelled by the recoil from the continuous evaporation of its top surface region (exactly by the pressure difference between the top and bottom surfaces of the protrusion) [27], this small protrusion keeps running down along the front keyhole wall. Here, the capillary force and Marangoni convection (i.e., thermal capillary force) also exist at the front keyhole wall to affect the flow of the protrusion. However, they are usually orders of magnitude smaller than the recoil pressure [28-30]. Therefore, under the present keyhole condition, the downflow of the protrusion is predominately controlled by the recoil pressure. The downflow speeds of the small protrusions, projected value on the imaging plane, are measured through the displacements of the protrusion centers, and their statistical distribution (a total of 182 objects in multiple spattering processes) is plotted in the inset of Fig. 2(c), with the mode value of $15 \mathrm{~m} / \mathrm{s}$. The recoil pressure, on one hand, is proportional to the saturated vapor pressure, which is determined by the melt's surface temperature [31,32], and on the other hand, it is related to the dynamic pressure of the melt, which is dependent on the square of the melt speed [32]. Hence, given the mode speed of $15 \mathrm{~m} / \mathrm{s}$, the average temperature on the front keyhole wall is estimated to be approximately $3800 \mathrm{~K}$ (see Appendix B 1), hundreds of kelvin higher than the boiling point of Ti-6Al-4V at $1 \mathrm{~atm}\left(T_{b}=3315 \mathrm{~K}\right.$ [33]). As a comparison, we also implement a one-dimensional (1D) steady-state model to derive the keyhole surface temperature as a function of the laser absorption intensity. Details of the model are provided in Appendix B1 and the Supplemental Material [6]. When considering the incident-angle-dependent absorption (aka Fresnel absorption) [26], the local temperature on the evaporation site of the front keyhole wall with a higher absorption intensity (e.g., on the small protrusions) could be even higher than $3800 \mathrm{~K}$, as shown in the Supplemental Material Fig. S8(a) [6].

The small protrusion at the front keyhole wall can partially reflect the incident laser upwards, and thus, the region underneath it receives less laser energy deposition, resulting in a local pressure drop around the keyhole bottom. With insufficient support from the recoil momentum on the keyhole side, the surrounding melt liquid starts filling the bottom, driven by surface tension. At the time $t_{1}$, the keyhole morphology changes into a reverse-trianglelike shape and is marked as RTS in Fig. 2(a). The formation of this unique shape can be attributed mainly to the first Fresnel absorption [26]. As depicted in the schematic, besides the front wall, the rear wall directly exposes its bottom region to the incident laser. Through the selfadjustments of the front and rear wall slopes, the local vapor plume pressures from the two walls tend to achieve a transient equilibrium at the keyhole bottom. We measure the keyhole depth over time and the result is shown in Fig. 2(c). Along with Video S2 in the Supplemental Material [6], one can find that most of the local minima below a depth threshold (e.g., $170 \mu \mathrm{m}$ ) in Fig. 2(c) correspond to the formation of this kind of reversetriangle-like keyhole. Statistically, under the current processing conditions, the depth of the reverse-trianglelike keyholes is measured to be $170 \pm 15 \mu \mathrm{m}$, about $35 \mu \mathrm{m}$ lower than the average keyhole depth of $205 \pm 15 \mu \mathrm{m}$.

Note here that the reverse-triangle-like keyhole is not perfectly symmetric with respect to the laser beam axis. A larger portion of the incident beam still impinges on the front keyhole wall, and the front wall is less steep than the rear wall. If considering the first Fresnel absorption, the effective laser absorption on the front wall could be higher, which is prone to causing a stronger evaporation. To balance the energy and the pressure, a stronger thermal conduction is expected at the front keyhole wall.

\section{Formation of a tonguelike protrusion and its bulk explosion}

Compared to the small dome-shaped protrusions in event no. 01 , the following protrusion in event no. 02 shows two distinct features: (i) the protrusion transforms into a rod shape, slightly tilting upward, and (ii) the protrusion size increases rapidly and collapses within $1 \mu \mathrm{s}$. As illustrated at the time of $t_{1}$ in Fig. 2(a), since the rear wall of the reversetriangle-like keyhole directly exposes its bottom to the incident laser beam, the generated metal vapor plume travels upwards towards the front wall. Squeezed by the recoil pressure at the upper surface and the dynamic vapor plume pressure at the lower surface, the protrusion changes from a dome shape slightly leaning downward to a rod shape tilting upward. From $t_{1}$ to the time when the detectable transition starts, we measure the distance between the bottom of the reverse-triangle-like keyhole and the bottom edge of the protrusion and roughly estimate the average travel speed of the vapor plume to be approximately $100 \mathrm{~m} / \mathrm{s}$ [19]. With the support of this directional vapor collision, the downflow of the protrusion is retarded, and its size starts to grow as the laser continues to advance. Shortly, a minikeyhole forms on the top of the protrusion. The multiple reflections and Fresnel absorption are prone to focusing the laser intensity at the minikeyhole bottom, producing an intensive evaporation and correspondingly a strong local recoil. Meanwhile, as the protrusion size increases, less laser energy is absorbed by the region underneath the protrusion, resulting in a drop of vapor plume pressure at the bottom. Therefore, the pressure difference on the upper and lower surfaces of this protrusion changes rapidly, driving it to swing down suddenly at time $t_{2}$ to become a tonguelike shape.

The unique geometry of this tonguelike protrusion largely increases the laser absorption. Figure 2(b) shows the distributions of absorption intensity on two different 
keyhole surfaces simulated using a ray-tracing model [30]. A detailed description of the simulation is documented in Appendix B 1 and the Supplemental Material [6]. The three-dimensional (3D) results are sectioned and rotated to highlight the absorption intensity distributions on the front keyhole walls. In the left panel of Fig. 2(b), a regular keyhole (without the tonguelike protrusion) exhibits nonuniform laser absorption and small wavy structures. In the right panel, a tonguelike protrusion is attached to the front keyhole wall, and the local absorption intensity under the same laser conditions can reach $5.0 \mathrm{MW} / \mathrm{cm}^{2}$, about twice the case for a regular keyhole.

This rapid and intense laser heating on the upper surface of the tonguelike protrusion can largely suppress the effective thermal conduction towards the subsurface. Here, it takes less than $1 \mu \mathrm{s}$ from the formation of the tonguelike protrusion to its collapse. If we assume the thermal diffusion time is $1 \mu \mathrm{s}$, the diffusion length for Ti-6Al-4V can be calculated to be only approximately $3 \mu \mathrm{m}$ (see Appendix B 3). With such a short diffusion length, it is reasonable to believe that the heat primarily accumulates on the upper region of the tonguelike protrusion. In addition, the laser heating might be so fast that the surface evaporation cannot accommodate it [34-36]. For simplicity, the energy loss due to surface evaporation on the tonguelike protrusion is assumed to be on the same order as that on the regular front keyhole wall. Then, through the latent heat and the mass flux of evaporation [37], it is estimated to be $0.37 \mathrm{MW} / \mathrm{cm}^{2}$ (see Appendix B 3), which is much smaller than the absorbed laser intensity of $5.0 \mathrm{MW} / \mathrm{cm}^{2}$. Hence, we can assume that all the absorbed laser energy is consumed for heating up the top surface layer (approximately $3 \mu \mathrm{m}$ ) of the molten tonguelike protrusion. With this assumption, the temperature of this surface layer can then reach thousands of kelvin above the boiling point of Ti-6Al-4V (see Appendix B 3). This could well suggest that the surface region of the protrusion is superheated, and the liquid is in a metastable state.

Given the $80-\mu \mathrm{m}$ laser beam size and the $500-\mathrm{mm} / \mathrm{s}$ scanning speed, the maximum possible dwell time of the laser beam on the tonguelike protrusion (beam size/ scanning speed) is $160 \mu \mathrm{s}$, much longer than the lifetime of this protrusion (less than $10 \mu \mathrm{s}$ from formation to collapse). While its upper layer liquid is superheated, the lower region of the protrusion likely remains in the normal liquid state, since it is not directly heated by the laser and is beyond the absorption depth and thermal diffusion length. With the fast falling and thinning of the tonguelike protrusion and uneven local recoil pressures on surface, we speculate that the superheated and normal liquids manage to contact and mix vigorously [38-40]. The highly heterogeneous thermal structure then results in ultrafast mass and heat transfers and eventually causes a violent evaporation inside the protrusion. The rapid escape of the vapor then shatters the tonguelike protrusion into fine droplets, with their sizes possibly smaller than the current imaging resolution (i.e., $3 \mu \mathrm{m} /$ pixel), showing as chaotic features in the keyhole. We hereby refer to the vanishing of the tonguelike protrusion as a bulk explosion process. This explosion is a highly dynamic and subsurface phenomenon which has never been discovered before in either experimental or theoretical work.

\section{Formation of melt ligaments and other events induced by the bulk explosion}

As the tonguelike protrusion explodes, almost simultaneously the front and rear keyhole walls exhibit discernable structure changes at time $t_{3}$ defined in Fig. 2(a), including rapid curvature change, emergence of thin melt ligaments, and roughening of vapor-liquid interfaces.

Upon impact of the vapor plume and small droplets created by the explosion of the tonguelike protrusion, the local curvatures around the keyhole rims undergo a sharp positive-to-negative change, and a crater is created on each side at time $t_{3}$. This suggests that the local dynamic pressure of the mixture is higher than the local surfacetension pressure. In a few microseconds, as the dynamic pressure decays, the crater becomes shallower and vanishes in the end.

While the craters on the keyhole walls develop, thin melt ligaments emerge from the keyhole rims and rise at high speeds. The ligament on the rear keyhole rim, which is $50 \mu \mathrm{m}$ away from the center of the tonguelike protrusion, rises at an initial vertical speed of $35 \mathrm{~m} / \mathrm{s}$. The ligament on the front keyhole rim, which is $55 \mu \mathrm{m}$ away from the tonguelike protrusion, rises at a vertical speed of $20 \mathrm{~m} / \mathrm{s}$. Even though their distances from the tonguelike protrusion are similar, the initial vertical speeds of the two ligaments are dramatically different, which implies that the pressure created by the explosion distributes nonuniformly in space. We suspect that the fine droplets traveling towards the front keyhole rim could get vaporized by the direct laser heating or by the hot vapor ejecting from the front keyhole wall. Based on Bernoulli's equation [32] (see Appendix B 1), the initial dynamic pressures of the ligaments are estimated to be on the order of a few MPa.

The random roughening or wrinkling of the vapor-liquid interface, particularly around the rear keyhole rim, is other evidence for the bulk explosion of the tonguelike protrusion. Unlike the impact from a pure vapor on a keyhole wall, which creates a local cavity with a relatively smooth surface, the mixture of vapor and fine droplets has distinct dynamic pressure distributions (high dynamic pressure by the droplets and low dynamic pressure by the vapor). Therefore, when the mixture collides with the keyhole wall, it produces a rough liquid-vapor interface, like a splash. From the x-ray images in Fig. 1, it can be observed that, compared to the rear keyhole rim, the vapor-liquid interface 
around the front keyhole rim is relatively smooth (no obvious inflections). Again, this is likely due to the evaporation of fine droplets by the laser when they travel towards the front keyhole rim.

\section{Formation of an instant keyhole pore}

In the event KP shown in Fig. 1, a pore or cavity forms at the keyhole bottom while the tonguelike protrusion appears and explodes. This does not happen every time the spattering occurs. The relationship between the laser absorption and the front keyhole wall morphology could be used for qualitatively explaining its formation. At the time of $137.08 \mu \mathrm{s}$, with the existence of the tonguelike protrusion above, which absorbs and scatters a considerable amount of laser energy, the protrusion below it does not experience rapid collapse, and the local vapor pressure at the keyhole bottom decreases. Then, at $138.00 \mu \mathrm{s}$, squeezed by the recoil pressure at the upper surface and the dynamic vapor pressure at the lower surface, this lower protrusion collides with the rear keyhole wall, and a pore or cavity is then generated. After the explosion of the upper protrusion, the incident laser beam directly impinges on the melt liquid above the pore, so the pore vanishes once the laser drills through the melt. In short, the formation of the tonguelike protrusion on the front keyhole wall could possibly have a secondary effect, promoting the generation of keyhole pores.

\section{Internal adaptive mechanism for the tonguelike protrusion}

The time notes $t_{1}, t_{2}$, and $t_{3}$ were previously defined. At $t_{1}$, a reverse-triangle-like keyhole forms. At $t_{2}$, the protrusion near the top surface catastrophically falls to the horizontal center of the keyhole. At $t_{3}$, thin melt ligaments emerge and rise. The time difference $t_{2}-t_{1}$ is the formation time of the tonguelike protrusion, and $t_{3}-t_{2}$ can be understood as its action time. The sum of the formation time and the action time is then the lifetime of the protrusion.

Figure 2(d) shows the relationship between $t_{3}-t_{2}$ and $t_{2}-t_{1}$ for multiple spattering processes. Interestingly, they follow a positive trend: The longer the formation time, the longer the action time. The correlation between the formation and action times may be explained by an internal adaptive mechanism. Because of the nonuniform Fresnel absorption and various attenuation factors (i.e., plume, plasma, etc.) $[26,41]$, the effective laser energy absorbed by the sample fluctuates considerably over time and location. A higher laser absorption tends to generate a higher vapor flux and thus a higher vapor dynamic pressure, which then supports a shorter formation time of the tonguelike protrusion. On the other hand, a higher laser absorption results in a higher superheating and a stronger mass explosion, which then decreases the action time of the tonguelike protrusion.

\section{From ligamentation to spattering}

In this section, we study the motions of melt ligaments and spatters. Specifically, we correlate the spatter motions with the front keyhole wall morphology. In Appendix B 6 and the Supplemental Material [6], we statistically analyze the size and occurrence frequency of spatters as functions of the laser scanning speed, and the results are plotted in the Supplemental Material Figs. S5-S7 [6].

\section{Motion tracking and pressure-field analysis}

After the bulk explosion of the tonguelike protrusion, melt ligaments emerge at the keyhole rims and then rise, neck, and eventually break up into spatters. In the spattering process shown in Fig. 1, five detectable spatters are generated, and they are marked as SP01-SP05 in the last image. Their trajectories are tracked and summarized in Fig. 3(a). Note that the trajectories are projections on the plane normal to the incident $\mathrm{X}$-ray beam. These five spatters can be categorized into two groups: the three moving along the laser scanning direction as the forward-flying group (SP01-SP03) and the two in the opposite direction as the backward-flying group (SP04 and SP05). Different from the forward-flying spatters, which travel almost straight at nearly constant speeds after they detach from the melt pool, the backward-flying spatters exhibit curved trajectories and variable speeds near the keyhole. Beyond a certain distance from the keyhole, the trajectories of the backward-flying spatters become nearly straight.

The curved trajectories of the backward-flying spatters reflect the angle mismatch between the initial ejection directions and the force exerted on the spatters. As illustrated in Fig. 3(a), the front keyhole wall develops a slope under the scanning laser conditions [25]. The vapor plume is ejected in a primary direction normal to the inclined front keyhole wall towards the opposite direction of the scanning. For the case of deep and narrow keyholes, the vapor plume ejecting from the bottom region of the front keyhole wall collides with the rear keyhole wall other than escapes directly. Therefore, in the present case, we assume that only the vapor plume from the front wall near the sample surface escapes from the keyhole and contributes to the formation of a directional pressure field around the laser beam, as illustrated in Fig. 3(a). The front keyhole wall may partially reflect the laser beam to the rear wall and generate vapor plume that escapes towards the scanning direction. Since the forward-flying spatters do not alter their directions during their flights, we may argue that the dynamic pressure of the vapor plume (vapor pressure field) created by the rear keyhole wall is negligible.

Before the breakup process, the motions of the ligaments are mainly determined by the drag force, while after the spatters separate from the melt pool, the vapor plume around them controls their fly directions and accelerations, as we discuss above. The vertical and horizontal speeds of the ligaments and spatters as functions of time are measured 

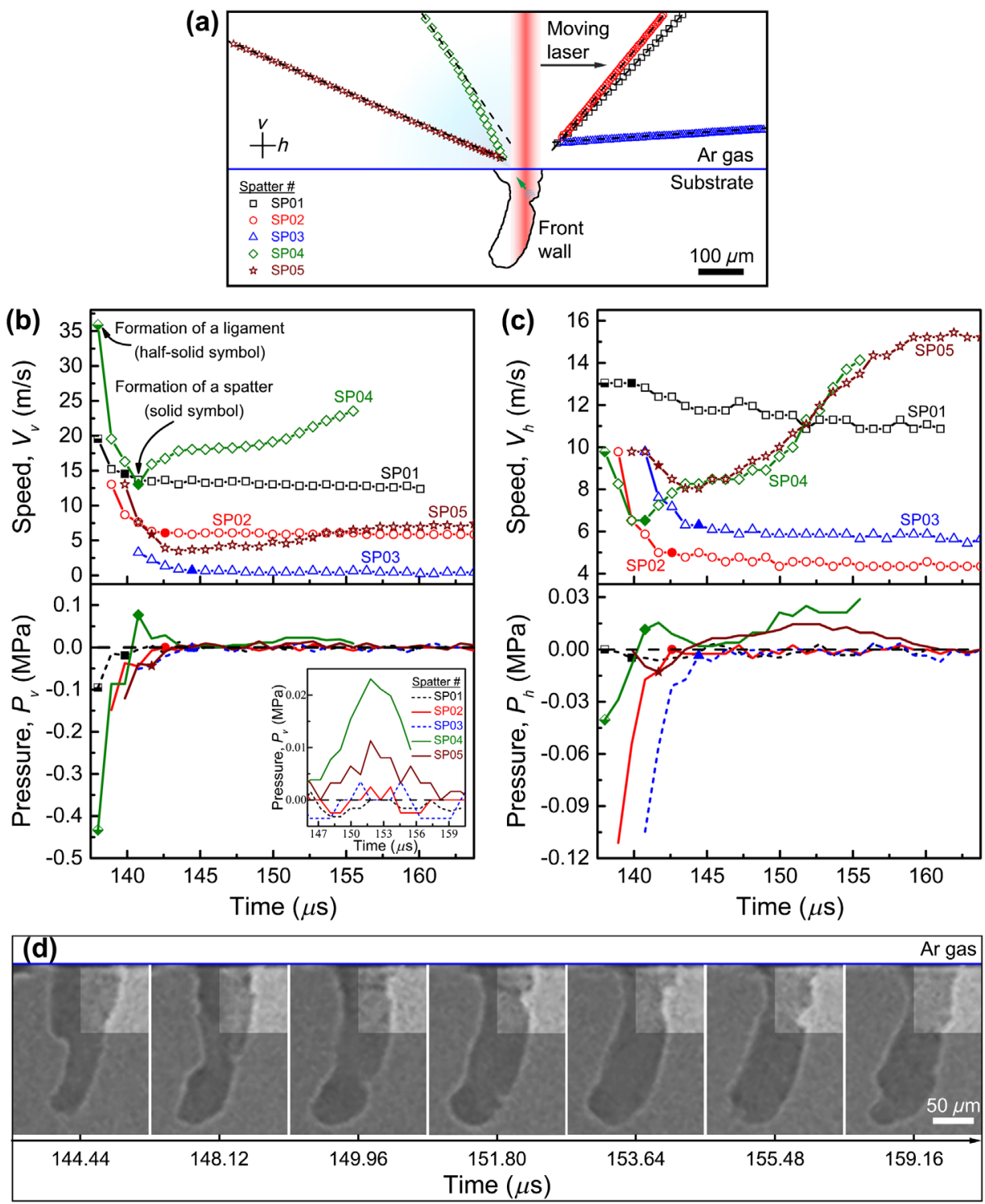

FIG. 3. Spatter behaviors and pressure field. (a) Trajectories of five ejected particles in one spattering process. SP01-SP03 are forward-flying spatters, and SP04 and SP05 are backward-flying spatters. The reference black dashed lines highlight the curved trajectories of the spatters SP04 and SP05. The diffused sky blue background on the left side of the laser indicates the vapor plume zone arising from the front wall of the keyhole. (b),(c) Vertical and horizontal travel speeds (upper panels) and the calculated pressures on melt ligaments and spatters (lower panels). The half-solid symbols in each plot indicate the times when the thin melt ligaments appear. The solid symbols mark the times when spatters separate from the melt pool. The negative pressure represents a net force that slows down the motion of the liquid structure, while the positive pressure accelerates the objects. The inset of (b) is a closer look at the vertical pressure field the spatters experience. (d) X-ray images of the keyhole morphology. The top region of the front keyhole wall is highlighted. The protrusion appearing between 149.96 and $155.48 \mu$ s increases the local laser absorption, creating a stronger vapor plume that is responsible for the increased pressures on spatters SP04 and SP05. All images shown here are background corrected using the images collected before the laser melting. The contrast is then reversed to highlight the keyhole dynamics.

from the x-ray videos and plotted in the upper panels of Figs. 3(b) and 3(c), respectively. The half-solid symbols in each plot correspond to the times when the melt ligaments appear, and the solid symbols indicate the times when spatters separate from the melt pool. We calculate the accelerations and the corresponding forces using Newton's law, $F=m a$. The average pressures the ligaments and spatters experience at different times are then obtained by dividing the forces by the cross-sectional areas of the spatters (see Appendix B 4). Here, for simplicity, the part of a ligament (the predecessor of a spatter) and the resulting spatter are assumed to have the same radius. The vertical and horizontal pressures are plotted in the lower panels of Figs. 3(b) and 3(c), respectively. Before the spatters 
separate from the melt pool, the pressures are generally negative, with the initial absolute value on the order of $10^{5} \mathrm{~Pa}$, suggesting that the drag force primarily slows down the motions of the ligaments. After the breakup, the pressures on the forward-flying spatters are close to zero, while the pressures on the backward-flying spatters are all positive, with the maximum value on the order of $10^{4} \mathrm{~Pa}$, indicating the vapor plume accelerates the spatters.

Surface tension is undoubtedly one of the main factors that holds the melt ligaments from breaking up. For Ti-6Al$4 \mathrm{~V}$, the surface-tension coefficient is $1.53 \mathrm{~N} / \mathrm{m}[42,43]$. If the radius of the necking region is $10 \mu \mathrm{m}$, the surfacetension pressure is then approximately $10^{5} \mathrm{~Pa}$ (see Appendix B 5), on the same order of the measured drag pressure upon the formation of a ligament. As for the contribution from the viscous dragging, we calculate the Reynolds number [44]. For a ligament with radius of $10 \mu \mathrm{m}$ and initial rising speed of approximately $10 \mathrm{~m} / \mathrm{s}$, the Reynolds number is on the order of 100 (see Appendix B 5), which indicates that the fluid flow is not a Stokes flow, so the viscous dragging is negligible.

\section{Influence of front keyhole morphology on spatter motions}

For the backward-flying spatters, after their separations from the melt pool, there is an interesting feature in their pressure-time plots: A peak can be observed at $t=$ $\sim 152 \mu$ s in the plots of spatters SP04 and SP05. This peak suggests a stronger vapor plume ejection at that time. To understand this phenomenon, x-ray images of the keyhole morphology evolution in this time window are examined. Clearly in Fig. 3(d), a protrusion forms at the front keyhole rim at $t=151.80 \mu \mathrm{s}$. As discussed earlier, the formation of a protrusion structure can considerably increase the local laser absorption. Therefore, the stronger evaporation from the upper surface of the protrusion creates a more intense vapor plume and thus a stronger vapor pressure field, which then drives the spatters within it (e.g., SP04 and SP05) to accelerate. After the protrusion flows down and vanishes, the vapor plume intensity reduces and so do the pressures and the spatter accelerations.

\section{DISCUSSION AND CONCLUSION}

In summary, we discover a novel mechanism for metal spattering under high-power-laser processing: The bulk explosion of a tonguelike protrusion forming on the front keyhole wall drives the melt ligamentation around the keyhole rims and the subsequent spattering. Using $\mathrm{MHz}$ single-pulse synchrotron-x-ray imaging, the complete physical processes involved in a spattering activity are captured with unprecedented detail. The transformation of the keyhole morphology from the typical J-like shape to a reverse-triangle-like shape creates directional vapor plume collisions towards the front keyhole wall, which serves as a necessary condition for the formation of a tonguelike protrusion. The dramatic and abrupt changes in the pressures on the protrusion drive it to catastrophically fall to the keyhole center, which, on one hand, largely increases the laser absorption on the top surface, and on the other hand, vigorously mixes the superheated and normal liquids within this protrusion. The irregular fluctuation in the internal thermal and pressure structures then triggers a process of bulk explosion shattering the tonguelike protrusion into a mixture of vapor and fine droplets. When the explosion lands at the rims of the keyhole walls, thin melt ligaments form, rise, neck, and eventually break up into spatters. The motion of a spatter is closely related to its surrounding vapor pressure field, which is mainly determined by the laser absorption of the front keyhole wall near the sample surface. The emergence of a protrusion structure at the front keyhole rim can produce an intense vapor plume and accelerate the spatters within the plume along their traveling directions.

Here, the identification of a bulk explosion is supported by the following evidence: (i) the dramatic increase in the laser absorptivity on the top surface of the tonguelike protrusion, (ii) the subsequent nearly synchronized responses from the front and rear keyhole walls (e.g., roughening of vapor-liquid interfaces around the keyhole rims), and (iii) the chaotic subresolution features in the keyhole. We attribute this bulk explosion phenomenon to the large thermal fluctuation inside the tonguelike protrusion. Superficially, it possesses some of the key characteristics of the well-defined phase explosion (aka explosive boiling) and vapor explosion (aka steam explosion and fuel coolant interactions) processes $[45,46]$.

In a phase explosion $[34,35,47,48]$ when the temperature approaches the critical temperature $\left(T_{c}\right)$, homogeneous nucleation occurs, and once the bubbles reach a critical radius, the metastable liquid decomposes into a mixture of gas and fine droplets instantly. This often occurs in a scenario involving high-power short-pulse lasers. In our case, it takes less than $1 \mu \mathrm{s}$ for the tonguelike protrusion to form and collapse, so the rapid heating can possibly suppress the heterogeneous nucleation and trigger a phase explosion. Even though our analytical calculation and 1D steady-state simulation do not suggest that the temperature of the upper surface of the tonguelike protrusion can reach the critical point of Ti-6Al-4V, we cannot simply rule out the possibility of phase explosion since our simple numerical models might neglect other important heating effects within the keyhole.

In a vapor explosion [38-40,49], the high-temperature and low-temperature liquids are coarsely separated by a vapor film of the low-temperature liquid. With the collapse of the vapor film by internal or external perturbation, the two liquids come in contact. The ultrafast heat transfer then promotes rapid vapor generation as well as melt fragmentation and atomization. Back to our case, the superheated 
and normal melts are initially separated because of the limited thermal diffusion. When the pressures on the tonguelike protrusion change abruptly, the catastrophic falling and thinning of the protrusion could possibly break the balance and trigger the explosion. Therefore, vapor explosion could also be responsible for the bulk explosion observed here.

A rigorous physical description of the bulk explosion remains a challenge. We do not have direct evidence that can attribute this phenomenon to either phase explosion or vapor explosion. However, our study reveals the need and directions for further developing the characterization techniques and numerical models. While direct thermal measurement could still be infeasible in the foreseeable future, the ongoing or planned constructions of diffraction-limited synchrotron facilities and high-repetition-rate free-electron lasers will enable ultrafast imaging with nanoscale resolution and frame rates beyond $100 \mathrm{MHz}$. The improved imaging capability will help capture more details of the explosion process (e.g., bubble generation). In terms of simulation, the current numerical models of laser-metal interaction are developed with major assumptions, even the most sophisticated 3D multiphysics models. For instance, the average temperature on the front keyhole wall is underestimated, and the plasma effect is often overlooked [50-53]. We believe experiments like ours can help calibrate some of the key material parameters used in simulation, and more importantly, encourage theorists to reevaluate the hypotheses and assumptions.

As our study reveals, laser spattering substantially connects with the keyhole stability. A small perturbation on the front keyhole wall morphology can alter the laser absorption and potentially induce sequential events that eventually lead to spattering. In laser welding, the community has recognized that the spattering is caused by the complex interplay of the melt flow and vapor plume, though how one factor affects the other has never been directly observed. A solution the community adopted is to feed a protective gas stream to the keyhole at a certain tilt angle $[2,14]$. In addition to preventing the metal from oxidation, another intention of this blowing gas is to open up and stabilize the keyhole in order to mitigate porosity generation and spattering. This strategy, however, will not work for laser-powder-bed additive manufacturing because the strong gas flow directly towards the keyhole will destroy the powder bed before it does any good.

In an electron-beam additive manufacturing process, the build chamber is maintained at elevated temperatures, and each layer of the powder bed is presintered by scanning the electron beam with a higher speed and/or a lower power than the build condition. Laser-powder-bed fusion usually does not apply presintering to reduce spattering, primarily because of the production cost. However, if spattering is the major factor that deteriorates the part quality, presintering to rigidize the powder bed is still a straightforward solution.
Another solution to mitigate spattering-induced defects is to introduce high-velocity laminar flow of protective gas above the powder bed. Such a gas flow intends to blow the spatters away from the build area and prevent them from falling back onto the powder bed. It has also been proven effective for improving the uniformity of laser absorption and thereby the repeatability of the printing process $[54,55]$. However, the laminar gas flow has very limited influence on the vapor plume dynamics inside the keyhole and the keyhole wall instabilities. Our study confirms the critical role of the melt and vapor flow in the laserspattering process. Unambiguously, spattering tends to happen when strong melt flow and intense vapor exist. Therefore, an effective approach to mitigate (if not exterminate) spattering is to suppress the melt flows around the keyhole. In practice, this could be achieved through innovations in laser-beam profiles and in-process feedforward control systems.

\section{ACKNOWLEDGMENTS}

The authors would like to thank Alex Deriy at the APS, and Zherui Guo and Wayne Chen at Purdue University for their assistance with the x-ray experiments. We also acknowledge Ali Erdemir at ANL, Jian Cao and Kornel Ehmann at Northwestern University, and Anthony Rollett at Carnegie Mellon University for the fruitful discussions. T. S., C. Z., N. P., and K. F. acknowledge the support from Laboratory Directed Research and Development funding from Argonne National Laboratory provided by the Director, Office of Science of the U.S. Department of Energy under Contract No. DE-AC02-06CH11357. L. C. would like to thank the National Science Foundation for their support (Grant No. 1762477). W. T. would like to thank the National Science Foundation for their support (Grant No. 1752218). This research uses resources of the Advanced Photon Source, a U.S. Department of Energy (DOE) Office of Science User Facility operated for the DOE Office of Science by Argonne National Laboratory under Contract No. DE-AC02-06CH11357.

\section{APPENDIX A: EXPERIMENTAL SYSTEMS}

\section{Materials and laser system}

Ti-6Al-4V plates (grade 23, Titanium Distribution Services Inc., USA) are cut into small pieces with a nominal dimension of $50 \mathrm{~mm}$ long $\times 3.0 \mathrm{~mm}$ high $\times 0.50 \mathrm{~mm}$ thick using electrical discharge machining. The samples are then polished using sandpaper, working all the way up to 1200 grit, and the final thickness is $0.39 \pm 0.01 \mathrm{~mm}$. In each experiment, a sample is loaded into a custom-built vacuum chamber, with the sample thickness along the $\mathrm{x}$-ray direction and the thickness centerline on the laser scanning plane. After the sample loading, the vacuum chamber is first pumped down using a mechanical pump below 500 mtorr and then purged with pure Ar gas to $1.0 \mathrm{~atm}$ (760 torr). In the 
experiments, no Ar gas flow is introduced inside the vacuum chamber. The laser system consists of a ytterbium fiber laser (IPG YLR-500-AC, USA) and a galvo laser scanner (intelli $S C A N_{\mathrm{de}} 30$, SCANLAB GmbH, Germany). The fiber laser is in single mode, providing pure Gaussian beam profiles. The wavelength and the maximum laser power are $1070 \mathrm{~nm}$ and $520 \mathrm{~W}$, respectively. Both continuous-wave $(\mathrm{CW})$ mode and pulsed mode (rate up to $50 \mathrm{kHz}$ ) are available. The scanner has a maximum scanning speed of $2.0 \mathrm{~m} / \mathrm{s}$. With a $\mathrm{f} / 340-\mathrm{mm}$ objective lens and a $\mathrm{f} / 85-\mathrm{mm}$ collimator, the laser beam size is $56 \mu \mathrm{m}\left(1 / e^{2}\right)$ at the focal plane. In the present experiment, the laser is operated in $\mathrm{CW}$ mode, the laser beam size is approximately $80 \mu \mathrm{m}\left(1 / e^{2}\right.$, approximately $2.0 \mathrm{~mm}$ defocusing below the focal plane), the laser power is $210 \mathrm{~W}$, and the laser scans in a single straight line at the speed of $500 \mathrm{~mm} / \mathrm{s}$.

\section{MHz single-pulse synchrotron-x-ray full-field imaging}

The $\mathrm{MHz}$ single-pulse synchrotron-x-ray imaging experiments are carried out at the 32-ID-B beam line of the Advanced Photon Source at the Argonne National Laboratory. A short-period undulator $(18 \mathrm{~mm})$ with the gap set to $12 \mathrm{~mm}$ is used to generate polychromatic $\mathrm{x}$ rays with the integrated flux of approximately $7 \times 10^{15} \mathrm{ph} / \mathrm{s}$ and the first harmonic energy at $24.3 \mathrm{kev}(\lambda=0.51 \AA)$. The imaging detection system consists of a $100-\mu$ m-thick $\mathrm{Lu}_{3} \mathrm{Al}_{5} \mathrm{O}_{12}$ : Ce scintillator, a $45^{\circ}$ reflection mirror, a $10 \times$ microscope objective lens $(\mathrm{NA}=0.28$, Edmund Optics Inc., USA), a tube lens, and a high-speed camera (HPV-X2, Shimadzu Corp., Japan). The pixel resolution is approximately $3 \mu \mathrm{m}$. The $\mathrm{x}$-ray images shown in this contribution are collected at a frame rate of $1.087 \times 10^{6}$ frames per second, and an effective exposure time of approximately $100 \mathrm{ps}$ (single x-ray pulse). The experiment is carried out when the APS operates in 24-bunch mode.

\section{APPENDIX B: QUANTITATIVE ANALYSIS}

\section{Average temperature on the front keyhole wall}

The recoil pressure $\left(P_{r}\right)$ bridges the protrusion speed $\left(v_{p}\right)$ and the average temperature on the front keyhole wall $\left(T_{s}\right)$ through a combination of Bernoulli's equation [32] $P_{r}=\frac{1}{2} \rho_{m} v_{p}^{2}$ and the saturated vapor pressure $\left(P_{s}\right)[31,32]$ $P_{r} \geq 0.55 \times P_{s}\left(T_{s}\right)=0.55 \times P_{0} \exp \left\{\left[\left(L_{v}\right) /\left(R_{v} T_{b}\right)\right]\right.$ $\left.\left(1-T_{b} / T_{s}\right)\right\}$, where $P_{0}$ is the ambient pressure, $T_{b}$ is the boiling temperature at $P_{0}, R_{v}$ is the gas constant, $L_{v}$ is the latent heat of evaporation, and $\rho_{m}$ is the mass density of the melt liquid. Hence, we can estimate the average temperature on the front keyhole wall from the protrusion speed by the equation $T_{s} \sim T_{b} /\left\{1-\left[\left(R_{v} T_{b}\right) / L_{v}\right] \ln \left[\left(\rho_{m} v_{p}^{2}\right) /\right.\right.$ $\left.\left.\left(1.1 P_{0}\right)\right]\right\}$. Here, for Ti-6Al-4V at $1 \mathrm{~atm}, T_{b}=3315 \mathrm{~K}$ [33], $P_{0}=1.013 \times 10^{5} \mathrm{~Pa}, R_{v}=8.314 \mathrm{~J} /(\mathrm{K} \mathrm{mol})$, which is converted into $181.25 \mathrm{~J} /(\mathrm{K} \mathrm{kg})$ through the molar mass of Ti-6Al-4V $(45.87 \mathrm{~g} / \mathrm{mol}), L_{v}=9.7 \times 10^{6} \mathrm{~J} / \mathrm{kg}$ [56], and $\rho_{m}=3920 \mathrm{~kg} / \mathrm{m}^{3}$ [42]. If the protrusion speed is taken as the mode value of $15 \mathrm{~m} / \mathrm{s}$, then the average temperature on the front keyhole wall is calculated to be approximately $3800 \mathrm{~K}$.

Another approach to estimate the keyhole surface temperature is to apply a 1D steady model [57]. The model considers a 1D configuration where a laser beam shines on a semi-infinite metal plate. The metal is first melted by the laser heating and then evaporation takes place to form a liquid-vapor interface. The calculation domain consists of a condensed phase (solid and liquid metal) and a gaseous phase (vapor metal and ambient air). When the steady state is reached, the pressure, velocity, and temperature on the vapor side of the liquid-vapor interface are denoted as $p_{v}, u_{v}$, and $T_{v}$; the pressure and temperature on the liquid side are denoted as $p_{l}$ and $T_{l}$. The model calculates the variables $\left(p_{v}, u_{v}, T_{v}, p_{l}, T_{l}\right)$ at the liquid-vapor interface given the laser absorption intensity $I_{a}$ as the model input. The results are shown in the Supplemental Material Fig. S8 [6].

\section{Laser-absorption intensity of a keyhole with and without the tonguelike protrusion}

Based on the multiple reflections and Fresnel absorption $[26,30,58]$, the ray-tracing technique is used to estimate the absorption intensity of a given keyhole shape extracted from the x-ray image frame with and without the tonguelike protrusion. First, artificial keyhole shapes are constructed based on experimental observations. Then, the laser beam is decomposed into discrete rays which are shot onto the artificial keyhole walls. Each ray contains a certain amount of power and can reflect multiple times on the keyhole surface. For each incidence of a ray on the keyhole surface, a certain portion of the ray power is absorbed at the incident location by the metal (absorptivity calculated by the Fresnel equation), and the reflected ray direction is calculated by the law of reflection. By tracing the reflections of each ray, a distribution of the absorbed laser intensity can be constructed on the keyhole surface. As shown in Fig. 2(b), on the tonguelike protrusion, the local absorption intensity is $5.0 \mathrm{MW} / \mathrm{cm}^{2}$.

\section{Average temperature on the upper layer of the tonguelike protrusion}

The thermal diffusion length $(L)$ can be estimated from the diffusivity of the melt liquid $(D)$ according to $L \sim \sqrt{D t_{d}}$, where $t_{d}$ is the diffusion time [59]. For Ti-6Al-4V, $D=$ $1.07 \times 10^{-5} \mathrm{~m}^{2} / \mathrm{s}$ [33]. If the diffusion time is approximately $1 \mu \mathrm{s}$, then the diffusion length is only approximately $3 \mu \mathrm{m}$. Therefore, for the tonguelike protrusion, the heat accumulates locally on the protrusion's upper side.

The energy loss due to surface evaporation $\left(Q_{v}\right)$ can be estimated from the latent heat $\left(L_{v}\right)$ and the mass flux of 
evaporation $(\dot{m})$ [37], $Q_{v}=-L_{v} \dot{m}$, where the mass flux $\dot{m}=\sqrt{m / 2 \pi k_{B} T} P_{s}, m$ is the atomic weight, $k_{B}$ is the Boltzmann constant, $T$ is the temperature, and $P_{s}$ is the saturated vapor pressure. For Ti-6Al-4V, $m \sim 47 \mathrm{u}$ (unified atomic mass unit, $1.66 \times 10^{-27} \mathrm{~kg}$ ) and $k_{B}=1.38 \times$ $10^{-23} \mathrm{~m}^{2} \mathrm{~kg} \mathrm{~s}^{-2} \mathrm{~K}^{-1}$. If the temperature is taken as the average temperature on the front keyhole wall, approximately $3800 \mathrm{~K}$, and the saturated vapor pressure is calculated from the Clausius-Clapeyron equation [31,32] $P_{s}=P_{0} \exp \left\{\left[L_{v} /\left(R_{v} T_{b}\right)\right]\left(1-T_{b} / T_{s}\right)\right\} \sim 7.9 \times 10^{5} \mathrm{~Pa}$, then the energy loss due to surface evaporation is approximately $0.37 \mathrm{MW} / \mathrm{cm}^{2}$. For rapid heating of the tonguelike protrusion, the surface vapor pressure cannot build up as rapidly $[34,35,47,48]$. Here we assume the energy loss due to surface evaporation is on the same order. Compared with the local absorption intensity on the upper layer of the tonguelike protrusion of $5.0 \mathrm{MW} / \mathrm{cm}^{2}$, it is negligible.

For simplicity, if we assume all the absorbed laser energy is consumed for locally heating up the tonguelike protrusion's upper layer, then through the energy balance [5,27], we have $I_{a} r_{p}^{2} \pi t_{a}=\rho_{m} V_{a} c_{m}\left(T-T_{m}\right)$, where $I_{a}$ is the absorption intensity, $r_{p}$ is the average radius of the tonguelike protrusion, $t_{a}$ is the duration of the laser absorption, $V_{a}$ is the volume of the melt liquid involved in the heating-up, $c_{m}$ is the heat capacity of the melt liquid, and $T_{m}$ is the melting point. Here, for Ti-6Al-4V, $r_{p} \sim 15 \mu \mathrm{m}, c_{m} \sim$ $830 \mathrm{~J} /(\mathrm{kg} \mathrm{K})$ [42], and $T_{m}=1928 \mathrm{~K}$ [33]. If the laser absorption intensity is taken from the ray-tracing simulation of approximately $5.0 \mathrm{MW} / \mathrm{cm}^{2}$, the duration of the laser absorption is approximately $0.5 \mu \mathrm{s}$ (the consequential explosion process occurs within one frame), the heating-up layer thickness is the thermal diffusion length $(L \sim 3 \mu \mathrm{m})$, and the involved volume is $L r_{p}^{2} \pi$ (assuming a cylinder shape). Then, the local temperature rise is estimated to be approximately $2600 \mathrm{~K}$, and the local temperature on the upper layer of the tonguelike protrusion is on the magnitude of approximately $4500 \mathrm{~K}$.

\section{Average pressure on a ligament or a spatter}

For simplicity, we assume that the predecessor of a spatter (part of a ligament) and the spatter have the same radius. Then, the corresponding accelerations $(a)$ and forces $(F)$ are estimated through $F=m a=\frac{4}{3} \pi r_{s}^{3} \times \rho_{m} \times[(\Delta v) /(\Delta t)]$, where $m$ is the mass of the spatter, $r_{s}$ is the radius, and $\Delta v$ is the speed change within the time duration of $\Delta t$. Furthermore, divided by the cross-sectional area of the spatter, we have the pressure $P_{s}=F / \pi r_{s}^{2}=\frac{4}{3} r_{s} \times \rho_{m} \times$ $[(\Delta v) /(\Delta t)]$.

\section{Dragging pressure caused by surface tension and viscosity}

The surface-tension pressure of a melt ligament is estimated through $P_{\text {surface }}=2 \gamma / r_{\text {lig }}$ [60], where $\gamma$ is the surface-tension coefficient, and $r_{\text {lig }}$ is the necking region radius. For Ti-6Al-4V, $\gamma \sim 1.53 \mathrm{~N} / \mathrm{m}[42,43]$. If the radius of the necking region is approximately $10 \mu \mathrm{m}$, the surfacetension pressure is approximately $10^{5} \mathrm{~Pa}$. The Reynolds number $(R e)$ is used to evaluate the contribution of the viscous force [44] $\mathrm{Re}=($ inertial force/viscous force $)=$ $\rho_{m} v_{s} L_{s} / \eta$, where $\eta$ is the viscosity of the melt, $L_{s}$ is the necking region diameter, and $v_{s}$ is the instant speed. For Ti-6Al-4V, $\eta \sim 0.005 \mathrm{Pas}$ [61]. If $L_{s}$ is approximately $20 \mu \mathrm{m}$ and $v_{s}$ is approximately $10 \mathrm{~m} / \mathrm{s}$, then the Reynolds number is on the order of 100 , which suggests that the viscous dragging is negligible.

\section{Statistical analysis of spatters}

With the laser beam size of approximately $80 \mu \mathrm{m}$, laser power of $210 \mathrm{~W}$, and scanning speed of $500 \mathrm{~mm} / \mathrm{s}$, we measure the sizes of 143 spatters and plot their size distribution in Fig. S5 of the Supplemental Material [6]. The mode size is $15 \mu \mathrm{m}$, and the maximum size is larger than $40 \mu \mathrm{m}$. Furthermore, under the same laser beam size and power, we vary the scanning speed in the range of 350 to $600 \mathrm{~mm} / \mathrm{s}$ and statistically analyze the influence of the scanning speed on the size and occurrence frequency of spatters. The occurrence frequency here is defined as the spatter number divided by the scanning length. In the Supplemental Material Fig. S6 [6], with the decrease of the scanning speed, the maximum spatter size increases rapidly and plateaus near $50 \mu \mathrm{m}$ when the scanning speed is below $350 \mathrm{~mm} / \mathrm{s}$. In the Supplemental Material Fig. S7 [6], the occurrence frequency of spatters drops linearly with the increase of the scanning speed, and it is extrapolated to approach zero when the scanning speed reaches $625 \mathrm{~mm} / \mathrm{s}$. More details and discussion are documented in the Supplemental Material [6].

[1] W. King, A. Anderson, R. Ferencz, N. Hodge, C. Kamath, S. Khairallah, and A. Rubenchik, Laser Powder Bed Fusion Additive Manufacturing of Metals; Physics, Computational, and Materials Challenges, Appl. Phys. Rev. 2, 041304 (2015).

[2] R. Fabbro, Melt Pool and Keyhole Behaviour Analysis for Deep Penetration Laser Welding, J. Phys. D 43, 445501 (2010).

[3] A. Kaplan and J. Powell, Spatter in Laser Welding, J. Laser Appl. 23, 032005 (2011).

[4] T. DebRoy and S. David, Physical Processes in Fusion Welding, Rev. Mod. Phys. 67, 85 (1995).

[5] S. Ly, A. M. Rubenchik, S. A. Khairallah, G. Guss, and M. J. Matthews, Metal Vapor Micro-Jet Controls Material Redistribution in Laser Powder Bed Fusion Additive Manufacturing, Sci. Rep. 7, 4085 (2017).

[6] See Supplemental Material at http://link.aps.org/ supplemental/10.1103/PhysRevX.9.021052 for Figs. S1S8, Table I, Videos S1-S2, and additional analyses. 
[7] C. Zhao, K. Fezzaa, R. W. Cunningham, H. Wen, F. Carlo, L. Chen, A. D. Rollett, and T. Sun, Real-Time Monitoring of Laser Powder Bed Fusion Process Using High-Speed X-Ray Imaging and Diffraction, Sci. Rep. 7, 3602 (2017).

[8] J. A. Slotwinski, E. J. Garboczi, P. E. Stutzman, C. F. Ferraris, S. S. Watson, and M. A. Peltz, Characterization of Metal Powders Used for Additive Manufacturing, J. Res. Natl. Inst. Stand. Technol. 119, 460 (2014).

[9] M. Simonelli, C. Tuck, N. T. Aboulkhair, I. Maskery, I. Ashcroft, R. D. Wildman, and R. Hague, A Study on the Laser Spatter and the Oxidation Reactions during Selective Laser Melting of 316L Stainless Steel, Al-Si10-Mg, and Ti-6Al-4V, Metall. Mater. Trans. A 46, 3842 (2015).

[10] C. L. A. Leung, S. Marussi, M. Towrie, R. C. Atwood, P. J. Withers, and P. D. Lee, The Effect of Powder Oxidation on Defect Formation in Laser Additive Manufacturing, Acta Mater. 166, 294 (2019).

[11] A. Bauereiß, T. Scharowsky, and C. Körner, Defect Generation and Propagation Mechanism during Additive Manufacturing by Selective Beam Melting, J. Mater. Process. Technol. 214, 2522 (2014).

[12] J. H. Martin, B. D. Yahata, J. M. Hundley, J. A. Mayer, T. A. Schaedler, and T. M. Pollock, 3D Printing of High-Strength Aluminium Alloys, Nature (London) 549, 365 (2017).

[13] C. L. A. Leung, S. Marussi, R. C. Atwood, M. Towrie, P. J. Withers, and P. D. Lee, In Situ X-Ray Imaging of Defect and Molten Pool Dynamics in Laser Additive Manufacturing, Nat. Commun. 9, 1355 (2018).

[14] K. Kamimuki, T. Inoue, K. Yasuda, M. Muro, T. Nakabayashi, and A. Matsunawa, Prevention of Welding Defect by Side Gas Flow and Its Monitoring Method in Continuous Wave Nd: YAG Laser Welding, J. Laser Appl. 14, 136 (2002).

[15] M. Zhang, G. Chen, Y. Zhou, S. Li, and H. Deng, Observation of Spatter Formation Mechanisms in HighPower Fiber Laser Welding of Thick Plate, Appl. Surf. Sci. 280, 868 (2013).

[16] Q. Guo, C. Zhao, L. I. Escano, Z. Young, L. Xiong, K. Fezzaa, W. Everhart, B. Brown, T. Sun, and L. Chen, Transient Dynamics of Powder Spattering in Laser Powder Bed Fusion Additive Manufacturing Process Revealed by In-Situ High-Speed High-Energy X-Ray Imaging, Acta Mater. 151, 169 (2018).

[17] B. Lane, S. Moylan, E. P. Whitenton, and L. Ma, Thermographic Measurements of the Commercial Laser Powder Bed Fusion Process at NIST, Rapid Prototyp. J. 22, 778 (2016).

[18] M. J. Matthews, G. Guss, S. A. Khairallah, A. M. Rubenchik, P. J. Depond, and W. E. King, Denudation of Metal Powder Layers in Laser Powder Bed Fusion Processes, Acta Mater. 114, 33 (2016).

[19] P. Bidare, I. Bitharas, R. Ward, M. Attallah, and A. Moore, Fluid and Particle Dynamics in Laser Powder Bed Fusion, Acta Mater. 142, 107 (2018).

[20] H. Zheng, H. Li, L. Lang, S. Gong, and Y. Ge, Effects of Scan Speed on Vapor Plume Behavior and Spatter Generation in Laser Powder Bed Fusion Additive Manufacturing, J. Manuf. Process. 36, 60 (2018).

[21] A. Matsunawa, J.-D. Kim, N. Seto, M. Mizutani, and S. Katayama, Dynamics of Keyhole and Molten Pool in Laser Welding, J. Laser Appl. 10, 247 (1998).
[22] A. Heider, J. Sollinger, F. Abt, M. Boley, R. Weber, and T. Graf, High-Speed X-Ray Analysis of Spatter Formation in Laser Welding of Copper, Phys. Procedia 41, 112 (2013).

[23] N. D. Parab, C. Zhao, R. Cunningham, L. I. Escano, K. Fezzaa, W. Everhart, A. D. Rollett, L. Chen, and T. Sun, Ultrafast X-Ray Imaging of Laser-Metal Additive Manufacturing Processes, J. Synchrotron Radiat. 25 (2018).

[24] R. Cunningham, C. Zhao, N. Parab, C. Kantzos, J. Pauza, K. Fezzaa, T. Sun, and A. D. Rollett, Keyhole Threshold and Morphology in Laser Melting Revealed by Ultrahigh-Speed X-Ray Imaging, Science 363, 849 (2019).

[25] R. Fabbro, M. Dal, P. Peyre, F. Coste, M. Schneider, and V. Gunenthiram, Analysis and Possible Estimation of Keyhole Depths Evolution, Using Laser Operating Parameters and Material Properties, J. Laser Appl. 30, 032410 (2018).

[26] A. Kaplan, A Model of Deep Penetration Laser Welding Based on Calculation of the Keyhole Profile, J. Phys. D 27, 1805 (1994).

[27] A. Matsunawa and V. Semak, The Simulation of Front Keyhole Wall Dynamics during Laser Welding, J. Phys. D 30, 798 (1997).

[28] J. Y. Lee, S. H. Ko, D. F. Farson, and C. D. Yoo, Mechanism of Keyhole Formation and Stability in Stationary Laser Welding, J. Phys. D 35, 1570 (2002).

[29] J. Kroos, U. Gratzke, and G. Simon, Towards a SelfConsistent Model of the Keyhole in Penetration Laser Beam Welding, J. Phys. D 26, 474 (1993).

[30] W. Tan, N.S. Bailey, and Y. C. Shin, Investigation of Keyhole Plume and Molten Pool Based on a ThreeDimensional Dynamic Model with Sharp Interface Formulation, J. Phys. D 46, 055501 (2013).

[31] S. I. Anisimov and V. A. Khokhlov, Instabilities in LaserMatter Interaction (CRC Press, Boca Raton, Florida, USA, 1995).

[32] V. Semak and A. Matsunawa, The Role of Recoil Pressure in Energy Balance during Laser Materials Processing, J. Phys. D 30, 2541 (1997).

[33] R. Rai, J. Elmer, T. Palmer, and T. DebRoy, Heat Transfer and Fluid Flow during Keyhole Mode Laser Welding of Tantalum, Ti-6Al-4V, 304L Stainless Steel and Vanadium, J. Phys. D 40, 5753 (2007).

[34] J. Shepherd and B. Sturtevant, Rapid Evaporation at the Superheat Limit, J. Fluid Mech. 121, 379 (1982).

[35] A. Miotello and R. Kelly, Laser-Induced Phase Explosion: New Physical Problems when a Condensed Phase Approaches the Thermodynamic Critical Temperature, Appl. Phys. A 69, S67 (1999).

[36] K. Goda, K. Tsia, and B. Jalali, Serial Time-Encoded Amplified Imaging for Real-Time Observation of Fast Dynamic Phenomena, Nature (London) 458, 1145 (2009).

[37] M. Courtois, M. Carin, P. Le Masson, S. Gaied, and M. Balabane, A New Approach to Compute Multi-Reflections of Laser Beam in a Keyhole for Heat Transfer and Fluid Flow Modelling in Laser Welding, J. Phys. D 46, 505305 (2013).

[38] S. Board, R. Hall, and R. Hall, Detonation of Fuel Coolant Explosions, Nature (London) 254, 319 (1975).

[39] M. Corradini, B. Kim, and M. Oh, Vapor Explosions in Light Water Reactors: A Review of Theory and Modeling, Prog. Nucl. Energy 22, 1 (1988). 
[40] E. Sher, T. Bar-Kohany, and A. Rashkovan, Flash-Boiling Atomization, Progr. Energy Combust. Sci. 34, 417 (2008).

[41] V. V. Semak, J. A. Hopkins, M. H. McCay, and T. D. McCay, A Concept for a Hydrodynamic Model of Keyhole Formation and Support During Laser Welding, in ICALEO ${ }^{\circledR}$ 1994: Proceedings of the International Congress on Applications of Lasers \& Electro-Optics, edited by $\mathrm{T}$. Dwayne McCay, A. Matsunawa, and H. Hügel (LIA, 1994), p. 641.

[42] K. C. Mills, Recommended Values of Thermophysical Properties for Selected Commercial Alloys (Woodhead Publishing, Cambridge, England, 2002).

[43] R. K. Wunderlich, Surface Tension, and Viscosity of Industrial Ti-Alloys Measured by the Oscillating Drop Method on Board Parabolic Flights, High temperature materials and Processes 27, 401 (2008).

[44] J. Eggers and E. Villermaux, Physics of Liquid Jets, Rep. Prog. Phys. 71, 036601 (2008).

[45] A. Hassanein, V. Belan, I. Konkashbaev, L. Nikandrov, V. Safronov, A. Zhitlukhin, and V. Litunovsky, Modeling and Simulation of Melt-Layer Erosion during Plasma Disruption, J. Nucl. Mater. 241, 288 (1997).

[46] G. Miloshevsky and A. Hassanein, Modeling of Macroscopic Melt Layer Splashing during Plasma Instabilities, J. Nucl. Mater. 415, S74 (2011).

[47] P. Lorazo, L. J. Lewis, and M. Meunier, Short-Pulse Laser Ablation of Solids: From Phase Explosion to Fragmentation, Phys. Rev. Lett. 91, 225502 (2003).

[48] N. Zhang, X. Zhu, J. Yang, X. Wang, and M. Wang, TimeResolved Shadowgraphs of Material Ejection in Intense Femtosecond Laser Ablation of Aluminum, Phys. Rev. Lett. 99, 167602 (2007).

[49] P. A. Quinto-Su, A Microscopic Steam Engine Implemented in an Optical Tweezer, Nat. Commun. 5, 5889 (2014).

[50] A. Pirri, R. Root, and P. S. Wu, Plasma Energy Transfer to Metal Surfaces Irradiated by Pulsed Lasers, AIAA J. 16, 1296 (1978).

[51] J. Dowden, P. Kapadia, and N. Postacioglu, An Analysis of the Laser-Plasma Interaction in Laser Keyhole Welding, J. Phys. D 22, 741 (1989).
[52] I. Miyamoto and K. Mori, Development of In-Process Monitoring System for Laser Welding, in ICALEO® 1995: Proceedings of the International Congress on Applications of Lasers \& Electro-Optics (LIA, 1995), p. 759.

[53] T. Maiwa, I. Miyamoto, and K. Mori, Properties of Keyhole Plasma in $\mathrm{CO}_{2}$ Laser Welding, in ICALEO ${ }^{\circledR}$ 1995: Proceedings of the International Congress on Applications of Lasers \& Electro-Optics (LIA, 1995), p. 708.

[54] B. Ferrar, L. Mullen, E. Jones, R. Stamp, and C. Sutcliffe, Gas Flow Effects on Selective Laser Melting (SLM) Manufacturing Performance, J. Mater. Process. Technol. 212, 355 (2012).

[55] A. Ladewig, G. Schlick, M. Fisser, V. Schulze, and U. Glatzel, Influence of the Shielding Gas Flow on the Removal of Process By-Products in the Selective Laser Melting Process, Addit. Manuf. 10, 1 (2016).

[56] W. Yan, W. Ge, Y. Qian, S. Lin, B. Zhou, W. K. Liu, F. Lin, and G. J. Wagner, Multi-Physics Modeling of Single/ Multiple-Track Defect Mechanisms in Electron Beam Selective Melting, Acta Mater. 134, 324 (2017).

[57] M. Aden, E. Beyer, and G. Herziger, Laser-Induced Vaporisation of Metal as a Riemann Problem, J. Phys. D 23, 655 (1990).

[58] B. J. Simonds, J. Sowards, J. Hadler, E. Pfeif, B. Wilthan, J. Tanner, C. Harris, P. Williams, and J. Lehman, TimeResolved Absorptance and Melt Pool Dynamics during Intense Laser Irradiation of a Metal, Phys. Rev. Applied 10, 044061 (2018).

[59] R. G. Pinnick, A. Biswas, R. L. Armstrong, S. G. Jennings, J. D. Pendleton, and G. Fernández, Micron-Sized Droplets Irradiated with a Pulsed $\mathrm{CO}_{2}$ Laser: Measurement of Explosion and Breakdown Thresholds, Appl. Opt. 29, 918 (1990).

[60] J. Volpp, Formation Mechanisms of Pores and Spatters during Laser Deep Penetration Welding, J. Laser Appl. 30, 012002 (2018).

[61] C. Körner, A. Bauereiß, and E. Attar, Fundamental Consolidation Mechanisms during Selective Beam Melting of Powders, Modelling Simul., Mater. Sci. Eng. 21, 085011 (2013). 